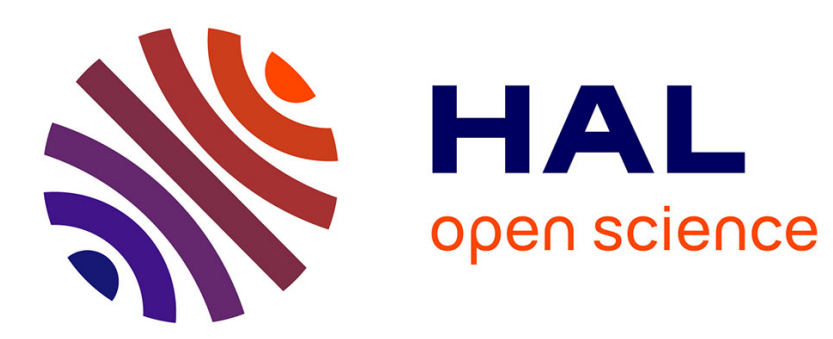

\title{
Multi-instrument Investigation of the Location of Saturn's Magnetotail X-Line
}

\author{
A. Smith, C. Jackman, M. Thomsen, L. Lamy, N. Sergis
}

\section{To cite this version:}

A. Smith, C. Jackman, M. Thomsen, L. Lamy, N. Sergis. Multi-instrument Investigation of the Location of Saturn's Magnetotail X-Line. Journal of Geophysical Research Space Physics, 2018, 123 (7), pp.5494-5505. 10.1029/2018JA025532 . hal-02300910

\section{HAL Id: hal-02300910 https://hal.science/hal-02300910}

Submitted on 29 Nov 2021

HAL is a multi-disciplinary open access archive for the deposit and dissemination of scientific research documents, whether they are published or not. The documents may come from teaching and research institutions in France or abroad, or from public or private research centers.
L'archive ouverte pluridisciplinaire HAL, est destinée au dépôt et à la diffusion de documents scientifiques de niveau recherche, publiés ou non, émanant des établissements d'enseignement et de recherche français ou étrangers, des laboratoires publics ou privés. 


\section{Journal of Geophysical Research: Space Physics}

\section{RESEARCH ARTICLE}

10.1029/2018JA025532

Key Points:

- Succession of reconnection products is inferred to come from three distinct $\mathrm{x}$-lines postdusk

- Short flow durations ( $\sim 10 \mathrm{~min}$ ) are the result of bursty or narrow $\left(\leq 4 R_{S}\right)$ $x$-lines

- Chain of dipolarizations identified postmidnight are inferred to come from a retreating $\mathrm{x}$-line (or $\mathrm{x}$-lines)

Correspondence to: A.W. Smith,

aw.smith@soton.ac.uk

Citation:

Smith, A. W., Jackman, C. M. Thomsen, M. F., Lamy, L., \& Sergis, N. (2018). Multi-instrument investigation of the location of Saturn's magnetotail $x$-line. Journal of Geophysical Research: Space Physics, 123, 5494-5505. https://doi.org/10.1029/2018JA025532

Received 28 MAR 2018 Accepted 18 JUN 2018 Accepted article online 25 JUN 2018 Published online 23 JUL 2018

(O2018. American Geophysical Union. All Rights Reserved.

\section{Multi-instrument Investigation of the Location of Saturn's Magnetotail X-Line}

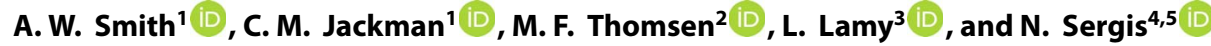 \\ ${ }^{1}$ Department of Physics and Astronomy, University of Southampton, Southampton, UK, ${ }^{2}$ Planetary Science Institute, \\ Tucson, AZ, USA, ${ }^{3}$ LESIA, Observatoire de Paris, CNRS, PSL, UPMC, UPD, Meudon, France, ${ }^{4}$ Office of Space Research and \\ Technology, Academy of Athens, Athens, Greece, ${ }^{5}$ Institute of Astronomy, Astrophysics, Space Applications and Remote \\ Sensing, National Observatory of Athens, Athens, Greece
}

Abstract Reconnection is a fundamentally important process in planetary magnetospheres, with both local and global effects. At Saturn, observations of the magnetotail reconnection site (or x-line) are rare, with only one in situ encounter reported to date. In this work, an extensive database of plasmoids and dipolarizations (Smith et al., 2016, https://doi.org/10.1002/2015JA022005) was investigated from a multi-instrument perspective in order to probe the location and variability of the magnetotail $x$-line. Several clear intervals were identified in which the $x$-line location could be indirectly inferred to move on relatively short timescales. Two case studies are presented, the first of which concerns short-lived flows, suggesting the reconnection sites can be either short-lived ( $\sim 10$ minutes) or extremely azimuthally limited ( $\sim 3 R_{S} / 0.4 \mathrm{hr}$ of local time). The second interval concerns the tailward motion of the reconnection site (or sites), inferred from the increasing electron temperature (and diminishing electron density) associated with the flows. This tailward motion occurs over $\sim 2.5 \mathrm{hr}$ (approximately a quarter of a planetary rotation). The composition of the suprathermal plasma suggests that this could be an example of the gradual depletion of mass-loaded flux tubes (that must occur prior to lobe reconnection). These case studies are consistent with previous statistical work that suggested that the site of reconnection in the Kronian magnetotail can be highly dynamic.

\section{Introduction}

Magnetic reconnection is an important physical process by which the energy stored in a magnetic field can be released. On a small scale it transfers energy stored in the magnetic field to the local plasma while reconfiguring the topology of the magnetic field. However, it can have global effects; reconnection on the dayside magnetopause can result in the addition of mass to a planetary magnetosphere. Within a planet's magnetotail it can result in the ejection of mass to the solar wind and potentially the closure of magnetic flux.

In a meridional picture of the magnetotail, oppositely directed field lines stretch down the tail. If these field lines come together and reconnect in several places, they create disconnected bubbles of plasma and field, known as plasmoids (Hones, 1976, 1977). A spacecraft located tailward of the reconnection sites may be expected to encounter these plasmoids as they are ejected down the magnetotail. At Saturn, a northward deflection of the field is expected to accompany a plasmoid observation (Jackman et al., 2007). The degree of deflection and accompanying changes in the other components of the field will depend on both the type of magnetic structure present (e.g., Figure 1 of Zong et al., 2004) and the relative trajectory of the spacecraft (e.g., Figure 1 of Borg et al., 2012). Meanwhile, planetward of the reconnection sites, the previously stretched field lines will contract, recovering to a more dipole-like arrangement and generating a dipolarization front (Nakamura et al., 2002; Ohtani et al., 2004; Runov et al., 2009). In the terrestrial magnetotail, the inward plasma flows associated with such fronts are observed over a large range of radial distances but are likely localized azimuthally (Gabrielse et al., 2014). At Saturn, the passage of the dipolarization front over the spacecraft is accompanied by a southward magnetic field rotation. Henceforth, plasmoids and dipolarizations may be referred to as reconnection products.

At Saturn, flows in the corotational direction (though below full corotational speed) are observed to dominate nightside magnetospheric convection (e.g., McAndrews et al., 2009; Thomsen, Jackman, et al., 2014), with only transient departures from the corotational direction observed. If a plasmoid is completely magnetically 
disconnected from the planet, it would be expected to move tailward and plasma outflow be observed. However, if the plasmoid is still trapped by closed field lines (that have yet to reconnect), then it will continue to be entrained with the ambient corotating plasma (Thomsen et al., 2013). Additionally, if the reconnection process does not expel the plasmoid such that its radial velocity is much greater than its azimuthal motion, then the flow will still have considerable momentum in the corotational direction. In a similar manner the corotational velocity may form a significant component of any inflow associated with a dipolarization.

At Saturn two global dynamic cycles could, in principle, operate. The first is the Dungey cycle (Dungey, 1961). This cycle can be thought of as beginning with the opening of magnetic flux on the dayside magnetopause via reconnection. Open field lines, mapping at one end to the planet and the other into the solar wind, are swept over the polar caps with the passage of the solar wind. Once within the magnetotail, they form the magnetotail lobes: regions above and below the plasma sheet sparsely populated by plasma. The open lobe field lines can subsequently close, with their ends mapping once more to both the northern and southern hemispheres, via reconnection at the center of the magnetotail. The cycle is completed as the now closed field lines circulate around the flanks to the dayside of the planet. This cycle dominates the magnetospheres of Earth and Mercury. At Saturn and at Earth, viscous-like interactions with the solar wind are also important (e.g., Delamere, Bagenal, et al., 2015, and references therein); Kelvin-Helmholtz interactions and resultant reconnection can act to transport mass, magnetic flux, and momentum across the magnetopause (e.g., Burkholder et al., 2017; Ma et al., 2017).

The second cycle is the Vasyliunas cycle (Vasyliunas, 1983), an internally driven process that results from the fast rotation of the planet and presence of significant internal mass sources, as are found at Jupiter and Saturn. Mass-laden flux tubes rotate around the dusk flank and are no longer supported by the magnetopause. The field lines stretch down the magnetotail, thinning the current sheet, eventually pinching off and reconnecting. At Saturn, the Vasyliunas cycle voltage is estimated to be several hundred kilovolts (Badman \& Cowley, 2007). In comparison, the Dungey cycle voltage has been estimated to range between $\sim 25 \mathrm{kV}$ during solar wind rarefactions and $\sim 150 \mathrm{kV}$ during compressions (Badman \& Cowley, 2007), with an average of $\sim 40 \mathrm{kV}$ (Masters, 2015). Therefore, in general, magnetotail reconnection can be expected to be predominantly Vasyliunas cycle-related. However, while the Kronian magnetosphere is strongly compressed, the Dungey cycle could contribute significantly to magnetotail dynamics (e.g., Thomsen et al., 2015).

A product of magnetotail reconnection (a dipolarization) was first identified within Saturn's magnetotail during Cassini's SOI (Saturn Orbit Insertion; Bunce et al., 2005). Since then numerous in situ encounters with plasmoids (Hill et al., 2008; Jackman et al., 2007, 2008; Russell et al., 2008) and dipolarizations (Jackman et al., 2013, 2015; Thomsen et al., 2013; Yao et al., 2017) have been observed. More recently, wider surveys of Saturn's magnetotail aiming to identify these structures have been conducted (e.g., Jackman et al., 2014; Smith et al., 2016, 2018; Thomsen, Jackman, et al., 2014).

The small region where magnetic fields reconnect is known as the diffusion region. The diffusion region can be divided into two characteristic scale sizes; the smaller electron diffusion region is surrounded by the larger ion diffusion region. At Earth, direct ion (Eastwood et al., 2010; Nagai et al., 2001; Øieroset et al., 2001) and electron (Burch et al., 2016) diffusion region encounters have been identified. However, at Saturn, only a single encounter with the magnetotail ion diffusion region has been reported (Arridge et al., 2015), and so modeling (Jia et al., 2012) or indirect evidence such as bursts of energetic ions (e.g., Mitchell et al., 2005) or the orientation of reconnection products (Smith et al., 2016, 2018) have been used to infer its location.

The situation is similar at Jupiter, where the location of the reconnection site has been inferred from the radial flow anisotropies (Kronberg et al., 2005, 2007; Woch et al., 2002) and the orientation of magnetic field deflections associated with reconnection products (Vogt et al., 2010). Overall, while a rough statistical separation has been found between planetward and tailward directed flows and structures (Woch et al., 2002; Vogt et al., 2010), individual Jovian events often show evidence of the dynamic nature of the reconnection site, e.g., $x$-line retreat (Kasahara et al., 2011; Kronberg et al., 2005). Likewise, at Saturn the location of the x-line has been inferred to be dynamic; indeed, this has precluded the estimation of a clear statistical $x$-line (Jackman et al., 2014; Smith et al., 2016). However, modeling efforts have suggested that Saturn's x-line is located between radial distances of $\sim 25$ and $40 R_{S}$, depending on both the upstream solar wind conditions and the current internal state (Jia et al., 2012). 
For this work an extensive catalog of reconnection products, identified from their magnetic signature (Smith et al., 2016), were investigated from a multi-instrument perspective to infer the location of the spacecraft relative to the magnetotail $x$-line. Section 2 describes the instrumentation used within the study. Section 3 presents two case studies, while section 4 discusses their implications in terms of $x$-line width and magnetospheric dynamics. Section 5 then summarizes and concludes.

\section{Instrumentation}

Data from the Cassini magnetometer (Dougherty et al., 2004) are used in this study. Magnetic field data are presented in Kronocentric-Radial-Theta-Phi coordinates: a planet-centered spherical polar system in which the radial component is directed outward from the planet, the theta (or meridional) component is positive southward and the azimuthal component is positive in the direction of planetary rotation (corotation). This coordinate system has been shown to be well suited to distinguish transient magnetospheric phenomena from periodic encounters with the magnetotail plasma sheet (Jackman, Arridge, et al., 2009).

The magnetic field data are complemented by the thermal electron and ion data from the Cassini Plasma Spectrometer (CAPS; Young et al., 2004). The Cassini Electron Spectrometer (ELS) observes the electron population between $0.58 \mathrm{eV}$ and $26 \mathrm{keV}$, while the lon Mass Spectrometer (IMS) measures ions with an energy per charge between $1 \mathrm{eV} / \mathrm{e}$ and $50 \mathrm{keV} / \mathrm{e}$. During most intervals the Cassini Plasma Spectrometer (CAPS) instrument does not see the full $4 \pi s r$ of the sky, unless the spacecraft happens to be rolling. Instead, an actuating platform allows sampling of $\sim 2 \pi s r$ of the sky. Electron moments (i.e., $n_{e}$ and $T_{e}$ ) derived from ELS data are also used, and the methods by which these moments are derived are described by Lewis et al. (2008) and Arridge, Gilbert, et al. (2009).

The thermal plasma data from CAPS are complemented by the suprathermal populations observed by the magnetospheric imaging instrument (Krimigis et al., 2004). In particular, data from the Charge Mass Spectrometer (CHEMS) are presented. CHEMS measures the mass and mass per charge of ions between 3 and $236 \mathrm{keV}$ for $\mathrm{H}^{+}$and between 8 and $236 \mathrm{keV}$ for $\mathrm{O}^{+}$. The collection of both mass and mass per charge information enables the separation of ion species such as $\mathrm{H}_{2}^{+}$and $\mathrm{He}^{++}$, an ability that is crucial to determining whether the plasma is of internal $\left(\mathrm{H}_{2}^{+}\right)$or external $\left(\mathrm{He}^{++}\right)$origin.

Finally, data from the Radio and Plasma Wave Science (RPWS; Gurnett et al., 2004) instrument provides a more global picture of the magnetospheric dynamics. The RPWS instrument allows monitoring of the Saturn kilometric radiation (SKR), typically observed between frequencies of $3 \mathrm{kHz}$ and 1.2 MHz (Kaiser \& Desch, 1984; Lamy et al., 2008).

\section{Results}

A catalog of reconnection products (Smith et al., 2016), identified from Cassini's magnetometer data, was investigated with data from multiple instruments in order to probe the distance of Cassini from the reconnection sites in Saturn's magnetotail. We present two case studies showing the motion or formation of magnetotail $x$-lines on short timescales. The first example shows the products of short-lived or azimuthally narrow $x$-lines on the dusk flank. The second example shows the tailward motion of an $x$-line (or series of $x$-lines) postmidnight over a quarter of a planetary rotation.

\subsection{Case Study 1: Premidnight Multiple X-Lines}

In this example, Cassini was approximately $33 R_{S}\left(1 R_{S}=60,268 \mathrm{~km}\right)$ from the planet on an inbound trajectory at $20: 00 \mathrm{hr}$ local time and a latitude close to the rotational equator $\left(0.12^{\circ}\right)$. It should be noted that the dipole equator is offset northward from the rotational equator by $\sim 0.04 R_{S}$ (Dougherty et al., 2005). Seven hours of Cassini Magnetometer and CAPS/ELS data are shown in Figure 1. Figure 2 shows CAPS/IMS viewing plots for the intervals highlighted (with corresponding letters above the axes) in Figure 1. The colored bars represent the orientation of the associated plasma flow relative to corotation (also shown in panel $\mathrm{g}$ ). The angle relative to the corotational direction $(\alpha)$ was calculated from plots such as those displayed in Figure 2: the angular separation between the peak of the observed counts and the corotational direction was evaluated. The parameter $\alpha$ is defined such that positive values indicate outflow, and it has been calculated for all times during which sufficient ion counts were recorded. Red bars indicate strong outflow $\left(\alpha>30^{\circ}\right)$, orange represents slightly weaker outflow $\left(20^{\circ}<\alpha<30^{\circ}\right)$ while yellow shows those within $20^{\circ}$ of corotation and thus fairly consistent with this, given uncertainties in the measurement (as in Thomsen et al., 2013). 


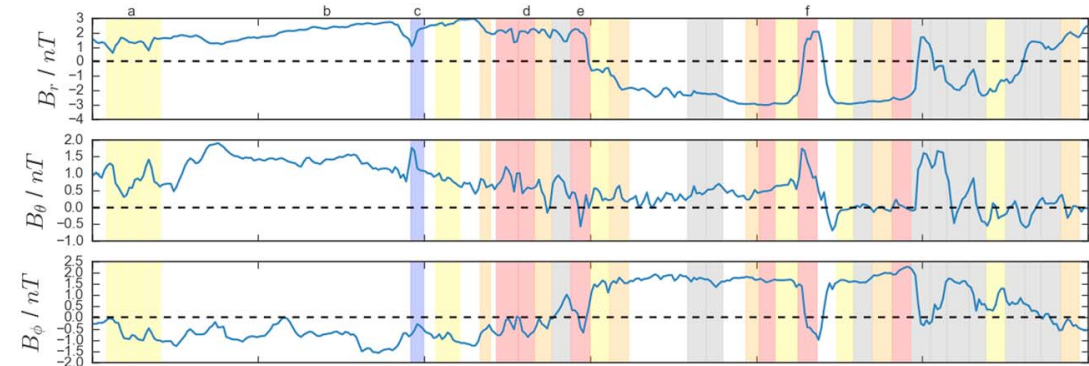

d
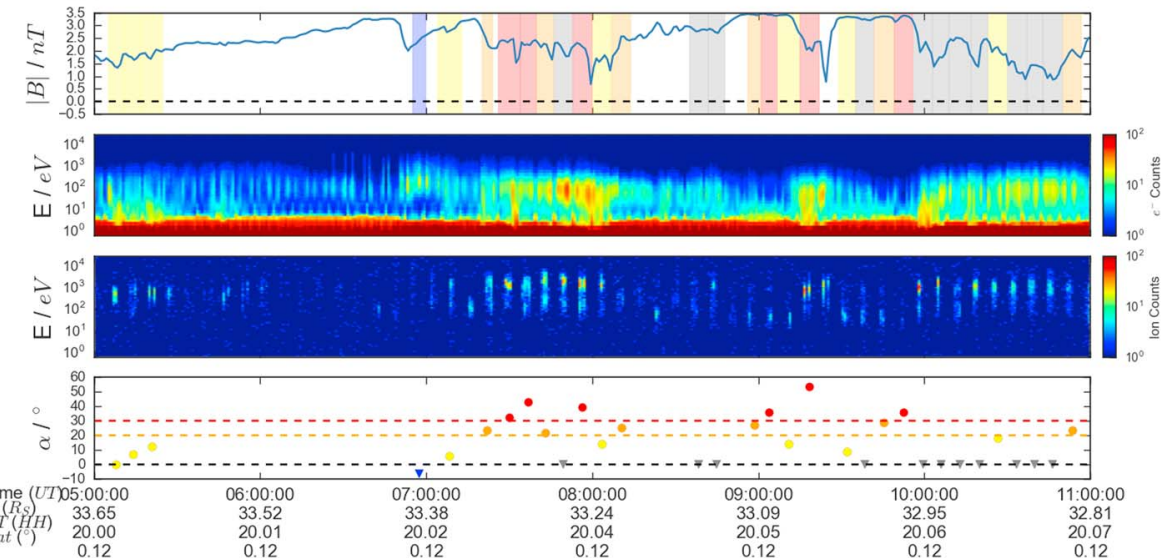

Figure 1. Seven hours of Cassini data from day 0402010 from 05:00 to 12:00 UT. Panels (a)-(d) shows 1-min resolution magnetometer data in the spherical polar Kronocentric-Radial-Theta-Phi coordinate system. Panel (e) shows a CAPS/ELS electron spectrogram, where the counts have been averaged over all anodes. Panel $(f)$ shows the ion spectrogram from anode 5, which generally showed the greatest counts during the interval. The vertical shaded regions outline the integration times used to produce the CAPS/IMS all-sky viewing plots, some of which feature in Figure 2. These intervals were analyzed to determine the angular separation between the peak of the counts and the direction of corotation $(\alpha)$, plotted in panel (g). Positive values of alpha represent outflow, while negative show inflow. During several intervals the peak of the flow was not observed within the field of view of CAPS/IMS, therefore only the derivation of upper limits were possible and are indicated with triangles in panel (g). Yellow regions (and points) show intervals where $\alpha$ was less than $20^{\circ}$, orange shows those between $20^{\circ}$ and $30^{\circ}$, while those for which $\alpha \geq 30^{\circ}$ are shown in red. The blue region shows an interval during which inflow can be inferred, while gray regions show those consistent with either inflow or corotation (i.e., the peak was not within the field of view). Six intervals are marked with letters at the top, which correspond to the relevant panels of Figure 2.CAPS/ELS = Cassini Plasma Spectrometer/Electron Spectrometer; IMS = Ion Mass Spectrometer.

Triangles indicate those intervals for which the peak flow is not within the field of view of the instrument, and therefore represent upper limits. The blue interval shows a time during which the region of sky just outside of corotation is visible and the peak is outside of this location (consistent with inflow), alternatively gray intervals show those for which the peak is not observed, but the instrument cannot confirm that the increasing counts continue beyond corotation.

At the start of the interval, between 05:00 and 05:30, the thermal plasma flows appear to be fairly corotational, consistent with previous statistical studies of the dominant flow patterns observed at Saturn (McAndrews et al., 2009; Thomsen et al., 2013). An example of an all-sky viewing plot for this configuration is presented in Figure 2a. Notably, the peak of the ion counts can be seen to coincide with the angle $90^{\circ}$ from the planetward direction, corresponding to an $\alpha$ of 0 (Figure 1g). Following this, between approximately 06:00 and 06:30, the radial component of the field increases while the temperature of the electron population appears to decrease. This could be indicative of field line stretching, perhaps as more heavily mass-loaded flux tubes have rotated around the dusk flank and are no longer supported by the magnetopause.

Shortly after 06:30 narrow vertical bars (indicating a highly anisotropic population) appear in the electron energy flux spectrogram at around $1 \mathrm{keV}$ (Figure 1e). These correspond to a population of energetic field-aligned electrons (confirmed in Figure 2b). Mitchell et al. (2009) inferred similar field-aligned distributions to be related to auroral currents. These electrons are followed at 06:50 by a southward rotation of the field in Figure $1 \mathrm{~b}$. This rotation of the field is accompanied by a small energization of the electron 

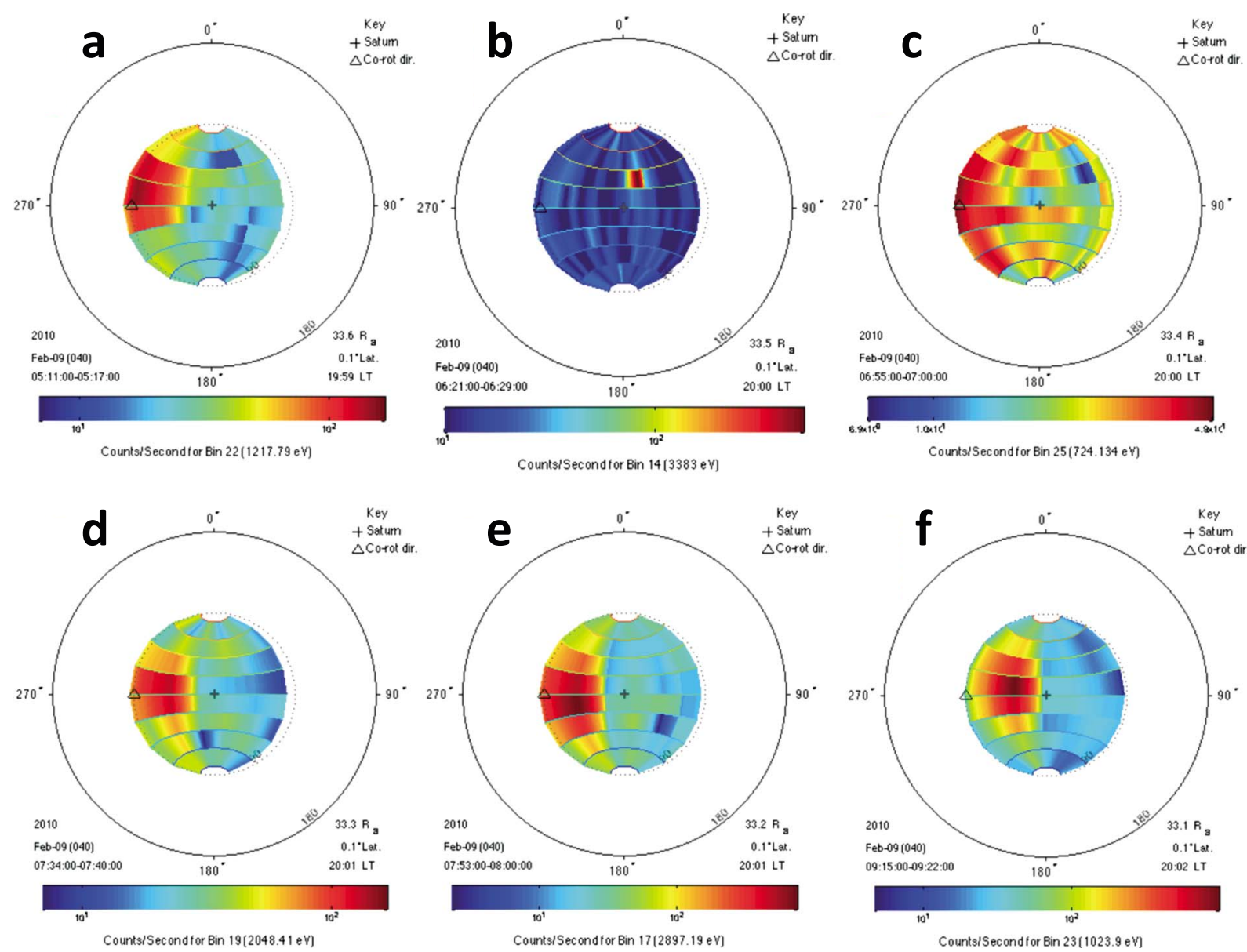

Figure 2. CAPS/IMS all-sky viewing plots (CAPS/ELS in panel b) detailing the origin of counts during the intervals defined at the top of Figure 1. The center of the plots represents the Saturnward looks direction (i.e., along the radial unit vector in the $-\hat{r}$ direction). The dotted circle represents directions a polar angle of $90^{\circ}$ from this, including the corotational direction (indicated with a triangle). The outer circle represents the anti-Saturnward direction. White regions show those areas of the full $4 \pi \mathrm{sr}$ not covered by the CAPS instrument during the given integration time (itself shown in the bottom left of each panel). The colors represent the count rate originating from that sector of sky. The CAPS/IMS energy bin used to produce the panel is provided at the bottom of each. CAPS/ELS $=$ Cassini Plasma Spectrometer/Electron Spectrometer; IMS = Ion Mass Spectrometer.

population: a signature that has been recently used to identify Kronian dipolarization fronts (Smith et al., 2018). Unfortunately, the CAPS instrument was oriented such that it was mostly looking in toward the planet at this time (hence, only in a position to detect plasma flowing outward from the planet and into the detector), and so cannot definitively confirm the presence of an inflowing plasma population. However, Figure $2 \mathrm{c}$ shows that the plasma counts increase toward the edge (left) of the field of view, extrapolating or fitting to this distribution would result in a flow originating from at least $5^{\circ}$ outside of corotation (shown by the blue triangle in Figure 1g). This would be consistent with the field rotation and small electron energization, indicating a dipolarization of the field. Following the dipolarization, the flow returns to something more indicative of corotation flow (shown by the yellow-shaded region and an $|\alpha|<5^{\circ}$ ). It is worth noting that inflow from dipolarizations may not be expected to travel radially inward at Saturn (as discussed in section 1); Figure $2 \mathrm{c}$ is consistent with an inflow originating between the anti-Saturnward (outer edge of the circle) and corotational direction (as discussed and observed by Thomsen et al., 2013). The duration of the inflow period was relatively short at $\sim 10 \mathrm{~min}$, shorter than the $30 \mathrm{~min}$ to several hour intervals previously reported at Saturn (e.g., Jackman et al., 2015; Thomsen et al., 2013, 2015).

Between approximately 07:30 and 07:50 a period of fairly significant outflow is observed (one of the intervals is displayed in Figure 2d). The azimuthal component of the magnetic field also changes sign, moving to a leading (or swept forward) configuration. Then, at 07:54, the field is deflected through $B_{\theta}=0$, briefly turning northward. This, coupled with observations that the plasma flow during this interval has a very significant 
a

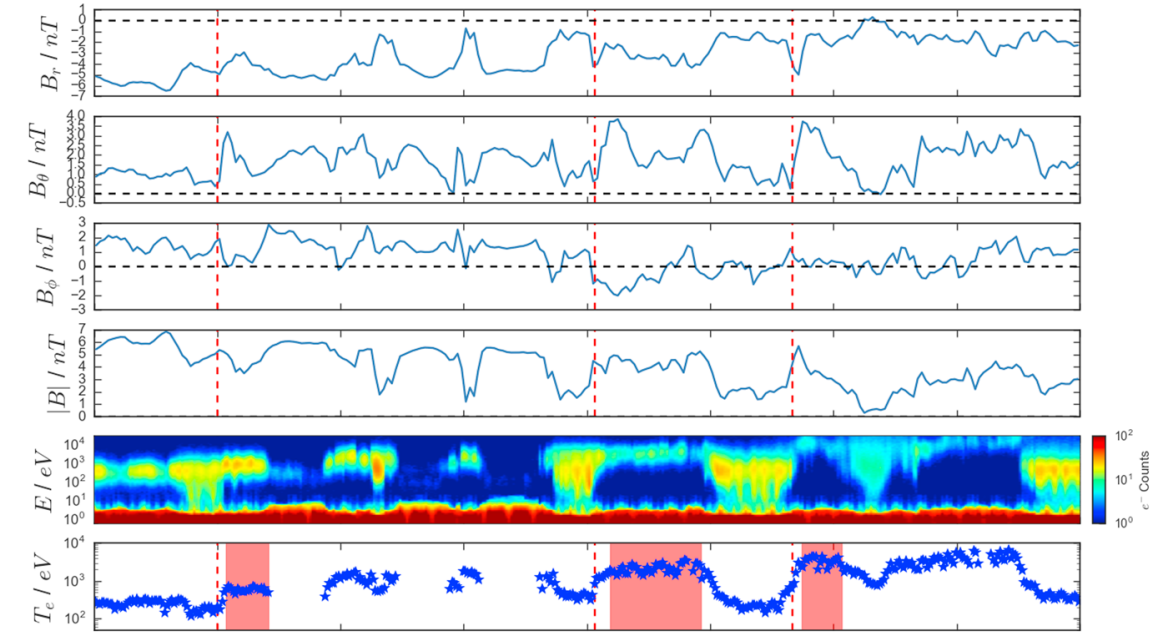

g

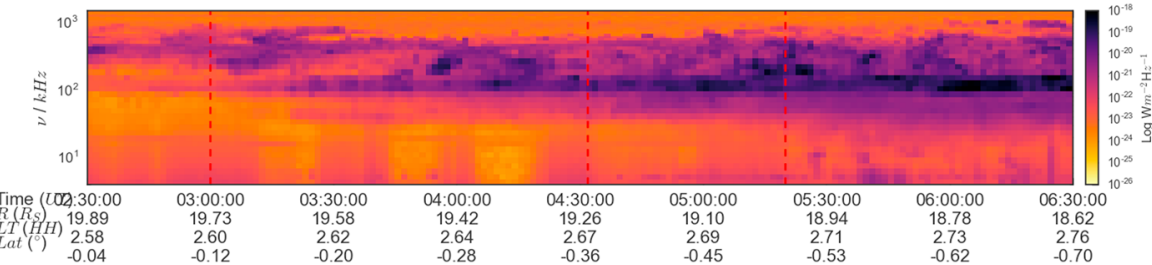

Figure 3. Cassini data from day 2272006 between 02:30 and 06:30 UT. Panels (a) - (e) are presented as in Figure 1. Panel (f) shows the electron temperature: calculated using the methods described by Lewis et al. (2008) and Arridge, Gilbert, et al. (2009). The red shading indicates the energetic/heated electron populations identified by Smith et al. (2018). Panel (g) displays a frequency-time spectrogram of the radio emissions from the Radio and Plasma Wave Science instrument.

outward component $\left(\alpha \sim 40^{\circ}\right)$ suggests that this could be the trailing edge of a plasmoid that has formed as the field has stretched downtail. The lack of a clear, preceding strong southward field deflection would indicate that the tailward hemisphere of the plasmoid is not encountered, or that the magnetic structure is not symmetric (e.g., Cowley et al., 2015). Though not a large field deflection ( $\Delta B_{\theta} \sim 1 \mathrm{nT}$ ) the outflow is significant compared to the adjacent measurements. The outflow is shown in Figure $2 \mathrm{e}$, where the peak of the ion counts can be seen to arrive from inward of the position of strict corotation (the hollow triangle). If this does represent a plasmoid, then an $\mathrm{x}$-line may have formed planetward (and duskward) of the spacecraft. Alternatively, the original $x$-line (associated with the dipolarization front) may have moved planetward, passing by the spacecraft which was located in the adjacent northern magnetotail lobe (from the large positive value of $B_{r}$ in this interval). The strong outflow and field deflection are only observed for a short period (during one 7-min integration for the outflow) before the flow angle reduces back toward corotation.

Just over an hour after the first plasmoid was observed, a second passed over the spacecraft, at around 09:15. The large, bipolar field deflection in $B_{\theta}$ is once more accompanied by outflow originating between the planetward and corotational directions (Figure 2f), as before. The return of the plasma toward corotational flow between the two plasmoids could indicate that this is due to a separate instance of reconnection, that an $x$-line has formed planetward and duskward of the spacecraft.

To summarize, in the space of just over $3 \mathrm{hr}$ (just under a third of a planetary rotation) we infer that Cassini observed three distinct products of reconnection. The first product, a dipolarization front, originating tailward of the spacecraft, while the later plasmoids formed between the spacecraft and the planet. The thermal plasma flows during the interval show a strong corotational influence but differ significantly from ambient azimuthal flow.

\subsection{Case Study 2: Postmidnight Tailward Motion}

For the second example Cassini was located postmidnight at around 02:30 hr local time, $\sim 20 R_{S}$ from the planet and latitudinally close to the rotational equator $\left(|\operatorname{Lat}|<1^{\circ}\right)$. Figure 3 shows $4 \mathrm{hr}$ of MAG data (Figures $3 a-3 d$ ) and thermal electron energy flux from CAPS/ELS (panel e). The derived electron temperature is presented in Figure $3 f$. 
a

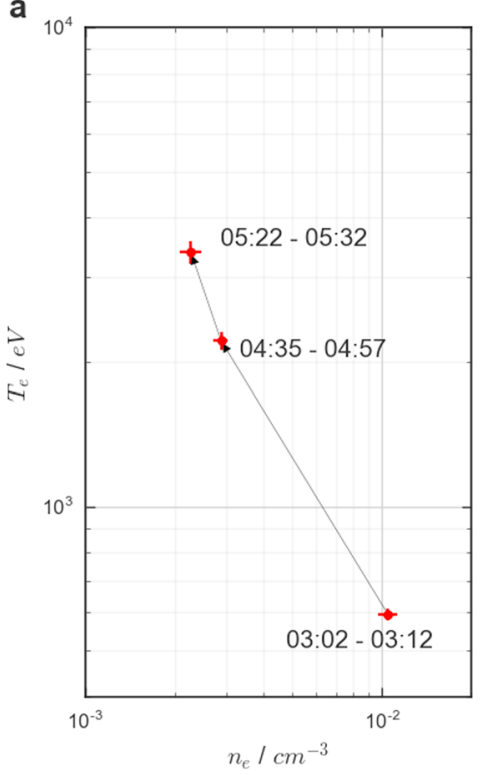

b

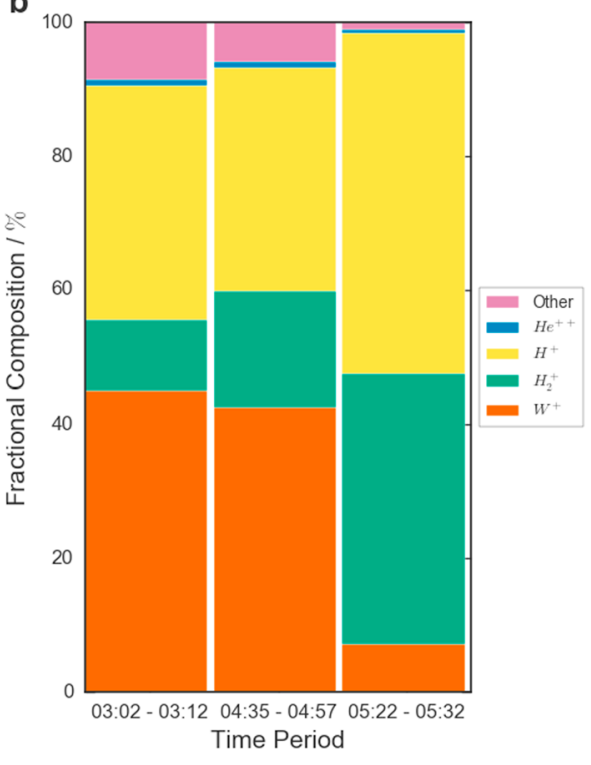

Figure 4. Cassini data summarizing the three dipolarizations in terms of a) electron temperature and density and b) suprathermal composition. Panel a displays the mean and standard error of the mean of the electron temperature and density moments (Arridge, Gilbert, et al., 2009; Lewis et al., 2008) within the heated regions (highlighted in Figure 3f). The suprathermal composition in panel $b$ is derived from the CHEMS instrument, integrated over the same integrals as above. CHEMS = Charge Mass Spectrometer.

Three clear dipolarizations are marked with vertical red dashed lines. These were identified based on the strong southward deflections (Figure $3 \mathrm{~b}$ ) and the change in electron population in Figure 3e. The change in electron population corresponds to an increase in the electron temperature and a reduction in the density of the dipolarizing material (cf. Smith et al., 2018). There are several other signatures present that could be dipolarizations (e.g. at 03:25 and 05:45); however they are not as clear as the three that are marked. The viewing of the CAPS instrument during this interval is purely inward, toward the planet and thus is unable to confirm the presence of an inflowing plasma population (or flow changes such as those in Figure 2).

Chains of dipolarizations are not uncommon in Saturn's magnetotail (Smith et al., 2016, 2018); however, this event is particularly significant as it shows a clear increase in the $T_{e}$ of the dipolarizing electron population with each subsequent dipolarization (highlighted with red shading in Figure $3 \mathrm{f}$ ). Before the first dipolarization the ambient plasma sheet has a temperature of $\sim 160 \mathrm{eV}$; this increases to around $600 \mathrm{eV}$ following the first dipolarization. The second and third dipolarizing populations then show average electron temperatures of $2.2 \mathrm{keV}$ and $3.4 \mathrm{keV}$, respectively. For context, Saturn's ambient (undisturbed) plasma sheet electron population is typically around $100 \mathrm{eV}$ (Arridge, McAndrews, et al., 2009). The $T_{e}$ change is shown in combination with the changing $n_{e}$ in Figure $4 a$.

The ion counts within the CAPS/IMS energy range (i.e., $E \leq 26 \mathrm{keV}$ ) are very low for these events, and so we turn to the magnetospheric imaging instrument suite of instruments to determine the composition of the dipolarizing material. CHEMS observes the suprathermal plasma population $(E \geq \sim 10 \mathrm{keV})$, which could differ from the thermal population if species-dependent heating or loss processes are significant. Figure $4 \mathrm{~b}$ shows the composition of the three heated intervals identified in Figure $3 \mathrm{f}$. The first two heated intervals (03:02-03:12 and 04:35-04:57) show markedly similar compositions, with $\mathrm{W}^{+}$group fractions of over $40 \%$ and very little $\mathrm{He}^{++}$. However, for the last interval (05:22-05:32) a strong fractional reduction in $\mathrm{W}^{+}$is observed, while $\mathrm{H}_{2}^{+}$and $\mathrm{H}^{+}$fractional abundances are seen to increase.

Figure $3 \mathrm{~g}$ shows the RPWS data during the interval of interest, allowing us a more global perspective of auroral dynamics. Shortly after 03:00 UT the intensity of the main band SKR emission increases (between approximately 100 and $400 \mathrm{kHz}$ (Kaiser \& Desch, 1984; Lamy et al., 2008)). This intensification is observed in both left- and right-handed polarizations (not shown) indicating emission from both northern and southern hemispheres. The emission continues to intensify throughout the plotted interval and gradually extends to lower frequencies (i.e., toward $\sim 10-20 \mathrm{kHz}$ around 06:00 UT). This characteristic change in the SKR is known as a 
low-frequency extension (LFE). In the past LFEs have been linked with both tail reconnection (Jackman, Lamy, et al., 2009; Lamy et al., 2013; Reed et al., 2018) and increases in solar wind dynamic pressure (Badman et al., 2008; Lamy et al., 2010). It should be noted that RPWS data are not included in Figure 1, as during that interval the radio intensity is much weaker.

\section{Discussion}

We now discuss the case studies with respect to their implications for magnetospheric dynamics.

\subsection{Case Study 1: Premidnight Multiple X-Lines}

Given the location of the spacecraft (postdusk) it is likely that the first example shows episodes of reconnection related to the Vasyliunas cycle (considering a flow regime comparable to Figure 2 of Cowley et al., 2004). As mass-laden flux tubes rotate around the dusk flank, they are no longer supported by the magnetopause and so can stretch down the magnetotail. As the flux tubes become stretched, the oppositely directed field lines either side of the magnetotail current sheet grow closer together, increasing the local gradient of the field and eventually leading to reconnection. The presence of significant thermal water group ions (of internal origin) in this interval supports this inference: this is the reconnection of closed flux.

The durations of the identified flows (10-30 $\mathrm{min}$ ) are less than the $30 \mathrm{~min}$ to several hours typically reported at Saturn (e.g., Arridge et al., 2015; Thomsen et al., 2013, 2015). These durations are much more in-line with the duration of the magnetic field signatures observed (e.g., Jackman et al., 2014; Smith et al., 2016). The short durations suggest (given the fast rotation of the Saturnian system) that either the $x$-lines are extremely limited in azimuth or that they form and disperse on very short timescales. A duration of $10 \mathrm{~min}$ and a velocity of $345 \mathrm{~km} / \mathrm{s}$ (the corotational plasma flow velocity at this radial distance, McAndrews et al., 2009), translates to a channel width of $3.4 R_{S}$ (or $\sim 0.4 \mathrm{hr}$ of local time). This value is likely to represent an upper limit; the typically observed (sub) corotational velocities at this radial distance are around $100-200 \mathrm{~km} / \mathrm{s}$ (McAndrews et al., 2009; Thomsen et al., 2013). This is, therefore, a relatively small-scale process compared to those previously observed. Small-scale, patchy reconnection was suggested for the Jovian magnetosphere by Bagenal (2007), and more recently Thomsen, Jackman, et al. (2014) and Delamere, Otto, et al. (2015) invoked a planetary wind or reconnection drizzle, respectively, on Saturn's dusk flank to explain their observations. The fact that the observed flows are not radial, and have a considerable corotational component suggests that the reconnection products formed at earlier local times. It has been suggested, from observations of northward magnetic field excursions on the dayside, that reconnection can initiate predusk in the dayside magnetosphere (Delamere, Otto, et al., 2015).

\subsection{Case Study 2: Postmidnight Tailward Motion}

The mechanisms that heat the plasma behind a dipolarization front are a combination of those local to the reconnection site itself (e.g., Fu et al., 2017; Phan et al., 2013) and the heating that occurs during the propagation of the front toward the planet, for example, adiabatic Fermi and betatron acceleration (e.g., Ashour-Abdalla et al., 2011; Birn et al., 2013, 2014; Fu et al., 2011; Pan et al., 2014) and nonadiabatic wave activity (e.g., Grigorenko et al., 2016) (nonlocal processes). At the terrestrial magnetopause, Phan et al. (2013) derived an empirical relation between the electron heating at the reconnection site $\left(\Delta T_{e}\right)$ and the Alfvén speed and plasma density of the reconnection inflow. Transposing the empirical relationship to Saturn's magnetotail requires extrapolation beyond the parameter space explored at the terrestrial magnetopause (due to the lower field strengths and plasma densities) and so should perhaps be interpreted with caution. However, using the measured lobe field strengths and the heated electron density (as a proxy for the inflowing population) leads to the conclusion that the local electron heating would be fairly significant (of the order of several hundred electron volts) but unable to explain the majority of the observed temperature change. Nonetheless, the empirical relationship suggests that a reduction in the inflowing plasma density would result in increased electron heating (Phan et al., 2013), though of an insufficient magnitude to fully explain the observations.

The majority of the electron heating can thus be attributed to further processing postreconnection (i.e., nonlocal heating effects). Therefore, as the magnitude of the heating increases significantly with subsequent events we could infer that the material has traveled a greater distance and that the site of reconnection is (or distinct sites are) receding deeper into the tail. Arridge et al. (2015) observed an interval during which Cassini passed through a tailward moving reconnection site, and therefore, this is perhaps a common occurrence. $X$-lines that retreat tailward are often observed at the Earth (e.g., Alexandrova et al., 2015; Eastwood et al., 2010) and also at Jupiter (Kasahara et al., 2011; Kronberg et al., 2005). Therefore, the second example likely shows an interval 
during which a reconnection site, or a series of reconnection sites, can be inferred to move down the magnetotail, farther from the spacecraft. Figure 4a shows that the subsequent events move up and to the left in density-temperature space: the increasing temperatures are accompanied by reducing densities.

It is also noteworthy that we are observing clear reconnection-related activity for a period of around $3 \mathrm{hr}$, almost a third of a planetary rotation. This suggests that the reconnection site/sites are either relatively stationary with respect to local time or are very widely extended (covering $\geq 7 \mathrm{hr}$ of local time).

The composition of the plasma can help determine whether the observed dipolarization is a result of the closure of open lobe field lines (and thus is Dungey cycle related) or purely involves closed field lines (and so is more likely part of the internally driven Vasyliunas cycle). It has been postulated that the closure of open field cannot occur until the equatorial, mass-loaded closed field has been reconnected and depleted (e.g., Thomsen et al., 2013).

For purely Vasyliunas cycle, closed field reconnection, it would be expected that the dipolarizing material would contain significant plasma of internal origin: including $\mathrm{H}^{+}, \mathrm{H}_{2}^{+}$, and heavier water group ions $\left(\mathrm{W}^{+}\right)$such as $\mathrm{O}^{+}, \mathrm{OH}^{+}, \mathrm{H}_{2} \mathrm{O}^{+}$, and $\mathrm{H}_{3} \mathrm{O}^{+}$. If the reconnection has proceeded to involve the closure of lobe field lines, then species of solar wind origin (such as $\mathrm{He}^{++}$) may be expected to be included. It should be noted that $\mathrm{H}^{+}$has both internal (e.g., Saturn's ionosphere and its moons) and external (i.e., the solar wind) sources and is thus not a good discriminator.

As mentioned above, the first two intervals (03:02-03:12 and 04:35-04:57) show similar (and predominantly internal) compositions, with over $40 \% \mathrm{~W}^{+}$group and little $\mathrm{He}^{++}$. However, the suprathermal count rate measured is markedly different, increasing from $10.4 \mathrm{~min}^{-1}$ to $56.7 \mathrm{~min}^{-1}$ (not shown). This could be a result of the plasma in the second event having been subject to greater heating, increasing the proportion of ambient plasma accelerated into the energy range of CHEMS ( $E \geq \sim 10 \mathrm{keV})$. The similar composition, and strong $\mathrm{W}^{+}$, is suggestive of the reconnection of closed field and therefore indicating they are likely Vasyliunas cycle related.

The final interval shows a strong reduction in $\mathrm{W}^{+}$group fraction; to less than $10 \%$. If this were indicative of open flux closure, then it may be expected that it would be replaced by $\mathrm{H}^{+}$and $\mathrm{He}^{++}$. However, instead we see the fractions of $\mathrm{H}_{2}^{+}$and $\mathrm{H}^{+}$increase. Due to the strong presence of $\mathrm{H}_{2}^{+}$, little if any lobe flux was closed during this event. Instead, it is possible that this event was associated with the reconnection of slightly higher latitude, more $\mathrm{W}^{+}$depleted field, and the release of a plasmoid. The plasmoid would act to preferentially remove the equatorial heavy $\mathrm{W}^{+}$group ions (Badman \& Cowley, 2007; Thomsen et al., 2013; Thomsen, Reisenfeld, et al., 2014). Though the $T_{e}$ of this event is the largest of the three events, the suprathermal count rate in this event reduces to $37.3 \mathrm{~min}^{-1}$ (from $56.7 \mathrm{~min}^{-1}$ in the previous dipolarizing interval). This could be explained by the reconnection occurring within a region of lower density, and thus, less plasma was available to be boosted into the suprathermal regime. Overall, this interval could be the in situ signature of the progression of reconnection to higher latitude, $\mathrm{W}^{+}$depleted flux tubes.

The field-aligned currents that stimulate the intensification of the SKR (and cause the LFE) are associated with the precipitation of $\sim 1$ - to 20-keV electrons into Saturn's atmosphere, producing UV (ultraviolet) aurora (Gustin et al., 2017; Lamy et al., 2010; Mutel et al., 2010; Tao et al., 2014). The link between magnetotail reconnection, LFEs, and auroral forms at Saturn was first postulated by Cowley et al. (2005). During SOI (Saturn Orbit Insertion), Bunce et al. (2005) observed the first event combining a magnetic field deflection, heated plasma (i.e., a dipolarization), and an LFE. Later, Jackman et al. (2013) investigated several dipolarizations, their associated field-aligned currents, and auroral imagery from an event inferred to be reconnection related. Unfortunately, no contemporaneous auroral imagery is available for the interval in the second case study; however, given both the in situ and RPWS data, it is likely that auroral emission would have been observed postmidnight (e.g., Lamy et al., 2013). In situ, the lack of species prevalent in the solar wind (e.g., $\mathrm{He}^{++}$) would suggest that the associated auroral emission would likely not approach the poleward boundary of the main oval (c.f. Nichols et al., 2014).

\section{Conclusions}

We have presented two examples of Saturn's magnetotail x-lines forming and moving on short timescales. In the first example three reconnection-related structures are observed, inferred to originate from at least two $x$-lines. The entire interval considered only spans around a third of a planetary rotation. The related thermal plasma flows only last for a small fraction of those previously reported (Arridge et al., 2015; 
Thomsen et al., 2013, 2015). This suggests that either the x-lines are extremely limited azimuthally $\left(\sim 3.4 R_{S}\right)$ or operate for very short intervals before dissipating, perhaps constituting reconnection drizzle as postulated by Delamere, Otto, et al. (2015). The interval is inferred to form a part of the Vasyliunas cycle as fresh, mass-laden flux tubes rotate around the dusk flank and reconnect. In particular, this event is in sharp contrast to that described by Thomsen et al. (2015), which represented a (rare) example of sustained lobe reconnection.

In the second example we infer the tailward motion of a magnetotail $x$-line or series of $x$-lines. The composition of the suprathermal plasma (greater than or equal to several kiloelectron volts) is not indicative of lobe reconnection during this interval and is instead suggestive of the shedding of $\mathrm{W}^{+}$-rich flux tubes that must precede reconnection of lobe flux. The interval is coincident with an intensification and lowering of the frequency of the SKR emission, likely related to the field-aligned currents generated by the magnetotail reconnection.

Acknowledgments

Part of this work was discussed within an ISSI team on "How does the Solar Wind Influence the Giant Planet Magnetospheres?" led by M. F. Vogt and A. Masters (ID: 357). A. W. S. thanks M. K. Dougherty for access to Cassini Magnetometer data, and S. Kellock and L. Alconcel at Imperial College London for MAG data processing. Cassini MAG data processing activities are supported in the United Kingdom by STFC. A. W. S. thanks A. J. Coates and G. R. Lewis for CAPS/ELS moments processing. A. W.S would like to thank C. S. Arridge, J. D. Nichols, D. G. Mitchell, T. D. Phan, and J. J. Reed for useful discussion. A. W. S. is funded by a SEPnet PhD studentship. C. M. J. is supported by a Science and Technology Facilities Council Ernest Rutherford FellowshipST/L004399/1. M. F. T. was supported by the NASA Cassini program through JPL contract 1243218 with Southwest Research Institute. L. L. was supported by CNES and CNRS PNP/PNST programs. The magnetic field and CAPS data shown in this analysis are available from the Planetary Data System (http://pds.nasa.gov/) The CAPS data are from volumes CO-E/J/S/SW-CAPS-3-UNCALIBRATED-V1.0 and $C O-E / J / S / S W-C A P S-5-D D R-E L E-$ MOMENTS-V1.0 (where the non-penetrating radiation temperatures were used). RPWS data are accessible from the online service http://www.lesia.obspm.fr/kronos/. Data analysis and plotting were conducted in Python and MATLAB. Specifically, the Python libraries used were NumPy, SciPy, Matplotlib, and Pandas.

\section{References}

Alexandrova, A., Nakamura, R., Semenov, V. S., \& Nakamura, T. K. M. (2015). Motion of reconnection region in the Earth's magnetotail. Geophysical Research Letters, 42(12), 4685-4693. https://doi.org/10.1002/2015GL064421

Arridge, C. S., Gilbert, L. K., Lewis, G. R., Sittler, E. C., Jones, G. H., Kataria, D. O., et al. (2009). The effect of spacecraft radiation sources on electron moments from the Cassini CAPS electron spectrometer. Planetary and Space Science, 57(7), 854-869. https://doi.org/10.1016/j.pss.2009.02.011

Arridge, C. S., McAndrews, H. J., Jackman, C. M., Forsyth, C., Walsh, A. P., Sittler, E. C., et al. (2009). Plasma electrons in Saturn's magnetotail: Structure, distribution and energisation. Planetary and Space Science, 57(14-15), 2032-2047. https://doi.org/10.1016/j.pss.2009.09.007

Arridge, C. S., Eastwood, J. P., Jackman, C. M., Poh, G.-K., Slavin, J. A., Thomsen, M. F., et al. (2015). Cassini in situ observations of long-duration magnetic reconnection in Saturn's magnetotail. Nature Physics, 12(3), 268-271. https://doi.org/10.1038/nphys3565

Ashour-Abdalla, M., El-Alaoui, M., Goldstein, M. L., Zhou, M., Schriver, D., Richard, R., et al. (2011). Observations and simulations of non-local acceleration of electrons in magnetotail reconnection events. Nature Physics, 7(4), 360-365. https://doi.org/10.1038/nphys1903

Badman, S. V., \& Cowley, S. W. H. (2007). Significance of Dungey-cycle flows in Jupiter's and Saturn's magnetospheres, and their identification on closed equatorial field lines. Annales Geophysicae, 25(4), 941-951. https://doi.org/10.5194/angeo-25-941-2007

Badman, S. V., Cowley, S. W. H., Lamy, L., Cecconi, B., \& Zarka, P. (2008). Relationship between solar wind corotating interaction regions and the phasing and intensity of Saturn kilometric radiation bursts. Annales Geophysicae, 26, 3641-3651.

Bagenal, F. (2007). The magnetosphere of Jupiter: Coupling the equator to the poles. Journal of Atmospheric and Solar-Terrestrial Physics, 69 , 387-402. https://doi.org/10.1016/j.jastp.2006.08.012

Birn, J., Hesse, M., Nakamura, R., \& Zaharia, S. (2013). Particle acceleration in dipolarization events. Journal of Geophysical Research: Space Physics, 118, 1960-1971. https://doi.org/10.1002/jgra.50132

Birn, J., Runov, A., \& Hesse, M. (2014). Energetic electrons in dipolarization events: Spatial properties and anisotropy. Journal of Geophysical Research: Space Physics, 119, 3604-3616. https://doi.org/10.1002/2013JA019738

Borg, A. L., Taylor, M. G. G. T., \& Eastwood, J. P. (2012). Annales geophysicae observations of magnetic flux ropes during magnetic reconnection in the Earth's magnetotail. Annales Geophysicae, 30(1992), 761-773. https://doi.org/10.5194/angeo-30-761-2012

Bunce, E. J., Cowley, S. W. H., Wright, D. M., Coates, A. J., Dougherty, M. K., Krupp, N., et al. (2005). In situ observations of a solar wind compression-induced hot plasma injection in Saturn's tail. Geophysical Research Letters, 32(20), L20S04. https://doi.org/10.1029/2005GL022888

Burch, J. L., Torbert, R. B., Phan, T. D., Chen, L.-J., Moore, T. E., Ergun, R. E., et al. (2016). Electron-scale measurements of magnetic reconnection in space. Science, 352(6290), aaf2939. https://doi.org/10.1126/science.aaf2939

Burkholder, B., Delamere, P. A., Ma, X., Thomsen, M. F., Wilson, R. J., \& Bagenal, F. (2017). Local time asymmetry of Saturn's magnetosheath flows. Geophysical Research Letters, 44, 5877-5883. https://doi.org/10.1002/2017GL073031

Cowley, S. W. H., Bunce, E. J., \& Prangè, R. (2004). Saturn's polar ionospheric flows and their relation to the main auroral oval. Annales Geophysicae, 22(4), 1379-1394. https://doi.org/10.5194/angeo-22-1379-2004

Cowley, S. W. H., Badman, S. V., Bunce, E. J., Clarke, J. T., Gerard, J. C., Grodent, D., et al. (2005). Reconnection in a rotation-dominated magnetosphere and its relation to Saturn's auroral dynamics. Journal of Geophysical Research, 110, A02201. https://doi.org/10.1029/2004JA010796

Cowley, S. W. H., Nichols, J. D., \& Jackman, C. M. (2015). Down-tail mass loss by plasmoids in Jupiter's and Saturn's magnetospheres. Journal of Geophysical Research: Space Physics, 120(8), 6347-6356. https://doi.org/10.1002/2015JA021500

Delamere, P. A., Bagenal, F., Paranicas, C., Masters, A., Radioti, A., Bonfond, B., et al. (2015). Solar wind and internally driven dynamics: Influences on magnetodiscs and auroral responses. Space Science Reviews, 187(1-4), 51-97. https://doi.org/10.1007/s11214-014-0075-1

Delamere, P. A., Otto, A., Ma, X., Bagenal, F., \& Wilson, R. J. (2015). Magnetic flux circulation in the rotationally-driven giant magnetospheres. Journal of Geophysical Research: Space Physics, 120, 4229-4245. https://doi.org/10.1002/2015JA021036

Dougherty, M. K., Kellock, S., Southwood, D. J., Balogh, A., Smith, E. J., Tsurutani, B. T., et al. (2004). The Cassini magnetic field investigation. Space Science Reviews, 114(1-4), 331-383.

Dougherty, M. K., Achilleos, N., Andre, N., Arridge, C. S., Balogh, A., Bertucci, C., et al. (2005). Cassini magnetometer observations during Saturn orbit insertion. Science, 307(5713), 1266-1270. https://doi.org/10.1126/science.1106098

Dungey, J. (1961). Interplanetary magnetic field and the auroral zones. Physical Review Letters, 6, 47-48. https://doi.org/10.1103/PhysRevLett.6.47.

Eastwood, J. P., Phan, T. D., Øieroset, M., \& Shay, M. A. (2010). Average properties of the magnetic reconnection ion diffusion region in the Earth's magnetotail: The 2001-2005 Cluster observations and comparison with simulations. Journal of Geophysical Research, 115, A08215. https://doi.org/10.1029/2009JA014962

Fu, H. S., Khotyaintsev, Y. V., Andre, M., \& Vaivads, A. (2011). Fermi and betatron acceleration of suprathermal electrons behind dipolarization fronts. Geophysical Research Letters, 38, L16104. https://doi.org/10.1029/2011GL048528

Fu, H. S., Vaivads, A., Khotyaintsev, Y. V., Andrè, M., Cao, J. B., Olshevsky, V., et al. (2017). Intermittent energy dissipation by turbulent reconnection. Geophysical Research Letters, 44, 37-43. https://doi.org/10.1002/2016GL071787

Gabrielse, C., Angelopoulos, V., Runov, A., \& Turner, D. L. (2014). Statistical characteristics of particle injections throughout the equatorial magnetotail. Journal of Geophysical Research: Space Physics, 119, 2512-2535. https://doi.org/10.1002/2013JA019638 
Grigorenko, E. E., Kronberg, E. A., Daly, P. W., Ganushkina, N. Y., Lavraud, B., Sauvaud, J. A., \& Zelenyi, L. M. (2016). Origin of low proton-to-electron temperature ratio in the Earth's plasma sheet. Journal of Geophysical Research: Space Physics, 121(10), 9985-10,004. https://10.1002/2016JA022874

Gurnett, D. A., Kurth, W. S., Kirchner, D. L., Hospodarsky, G. B., Averkamp, T. F., Zarka, P., et al. (2004). The Cassini radio and plasma wave investigation. Space Science Reviews, 114(1-4), 395-463. https://doi.org/10.1007/s11214-004-1434-0

Gustin, J., Grodent, D., Radioti, A., Pryor, W., Lamy, L., \& Ajello, J. (2017). Statistical study of Saturn's auroral electron properties with Cassini/UVIS FUV spectral images. Icarus, 284, 264-283. https://doi.org/10.1016/J.ICARUS.2016.11.017

Hill, T. W., Thomsen, M. F., Henderson, M. G., Tokar, R. L., Coates, A. J., Mcandrews, H. J., et al. (2008). Plasmoids in Saturn's magnetotail. Journal of Geophysical Research, 113, A01214. https://doi.org/10.1029/2007JA012626

Hones, E. W. (1976). The magnetotail - Its generation and dissipation. Physics of Solar Planetary Environments, 2, $558-571$.

Hones, E. W. (1977). Substorm processes in the magnetotail-Comments on 'On hot tenuous plasmas, fireballs, and boundary-layers in Earth's magnetotail'. Journal of Geophysical Research, 82(35), 5633-5640.

Jackman, C. M., Russell, C. T., Southwood, D. J., Arridge, C. S., Achilleos, N., \& Dougherty, M. K. (2007). Strong rapid dipolarizations in Saturn's magnetotail: In situ evidence of reconnection. Geophysical Research Letters, 34, L11203. https://doi.org/10.1029/2007GL029764

Jackman, C. M., Arridge, C. S., Krupp, N., Bunce, E. J., Mitchell, D. G., McAndrews, H. J., et al. (2008). A multi-instrument view of tail reconnection at Saturn. Journal of Geophysical Research, 113, A11213. https://doi.org/10.1029/2008JA013592

Jackman, C. M., Arridge, C. S., McAndrews, H. J., Henderson, M. G., \& Wilson, R. J. (2009). Northward field excursions in Saturn's magnetotail and their relationship to magnetospheric periodicities. Geophysical Research Letters, 36, L16101. https://doi.org/10.1029/2009GL039149

Jackman, C. M., Lamy, L., Freeman, M. P., Zarka, P., Cecconi, B., Kurth, W. S., et al. (2009). On the character and distribution of lower-frequency radio emissions at Saturn and their relationship to substorm-like events. Journal of Geophysical Research, $114, \mathrm{~A} 08211$. https://doi.org/10.1029/2008JA013997

Jackman, C. M., Achilleos, N., Cowley, S. W. H., Bunce, E. J., Radioti, A., Grodent, D., et al. (2013). Auroral counterpart of magnetic field dipolarizations in Saturn's tail. Planetary and Space Science, 82-83, 34-42. https://doi.org/10.1016/j.pss.2013.03.010.

Jackman, C. M., Slavin, J. A., Kivelson, M. G., Southwood, D. J., Achilleos, N., Thomsen, M. F., et al. (2014). Saturn's dynamic magnetotail: A comprehensive magnetic field and plasma survey of plasmoids and traveling compression regions and their role in global magnetospheric dynamics. Journal of Geophysical Research: Space Physics, 119, 5465-5494. https://doi.org/10.1002/2013JA019388

Jackman, C. M., Thomsen, M. F., Mitchell, D. G., Sergis, N., Arridge, C. S., Felici, M., et al. (2015). Field dipolarization in Saturn's magnetotail with planetward ion flows and energetic particle flow bursts: Evidence of quasi-steady reconnection. Journal of Geophysical Research: Space Physics, 120, 3603-3617. https://doi.org/10.1002/2015JA020995

Jia, X., Hansen, K. C., Gombosi, T. I., Kivelson, M. G., Tòth, G., Dezeeuw, D. L., \& Ridley, A. J. (2012). Magnetospheric configuration and dynamics of Saturn's magnetosphere: A global MHD simulation. Journal of Geophysical Research, 117, A05225. https://doi.org/10.1029/2012JA017575

Kaiser, M. L., \& Desch, M. D. (1984). Radio emissions from the planets Earth, Jupiter, and Saturn. Reviews of Geophysics, 22(4), 373-384. https://doi.org/10.1029/RG022i004p00373

Kasahara, S., Kronberg, E. A., Krupp, N., Kimura, T., Tao, C., Badman, S. V., et al. (2011). Magnetic reconnection in the Jovian tail: X-line evolution and consequent plasma sheet structures. Journal of Geophysical Research, 116, A11219. https://doi.org/10.1029/2011 JA016892

Krimigis, S. M., Mitchell, D. G., Hamilton, D. C., Livi, S., Dandouras, J., Jaskulek, S., et al. (2004). Magnetosphere imaging instrument (MIMI) on the Cassini mission to Saturn/Titan. Space Science Reviews, 114(1-4), 233-329. https://doi.org/10.1007/s11214-004-1410-8

Kronberg, E. A., Woch, J., Krupp, N., Lagg, A., Khurana, K. K., \& Glassmeier, K. H. (2005). Mass release at Jupiter: Substorm-like processes in the Jovian magnetotail. Journal of Geophysical Research, 110, A03211. https://doi.org/10.1029/2004JA010777

Kronberg, E. A., Glassmeier, K.-H., Woch, J., Krupp, N., Lagg, A., \& Dougherty, M. K. (2007). A possible intrinsic mechanism for the quasi-periodic dynamics of the Jovian magnetosphere. Journal of Geophysical Research, 112, A05203. https://doi.org/10.1029/2006JA011994

Lamy, L., Schippers, P., Zarka, P., Cecconi, B., Arridge, C. S., Dougherty, M. K., et al. (2010). Properties of Saturn kilometric radiation measured within its source region. Geophysical Research Letters, 37, L12104. https://doi.org/10.1029/2010GL043415

Lamy, L., Prangè, R., Pryor, W., Gustin, J., Badman, S. V., Melin, H., et al. (2013). Multispectral simultaneous diagnosis of Saturn's aurorae throughout a planetary rotation. Journal of Geophysical Research: Space Physics, 118, 4817-4843. https://doi.org/10.1002/jgra.50404

Lamy, L., Zarka, P., Cecconi, B., Prangè, R., Kurth, W. S., \& Gurnett, D. A. (2008). Saturn kilometric radiation: Average and statistical properties. Journal of Geophysical Research, 113, A07201. https://doi.org/10.1029/2007JA012900

Lewis, G. R., Andrè, N., Arridge, C. S., Coates, A. J., Gilbert, L. K., Linder, D. R., \& Rymer, A. M. (2008). Derivation of density and temperature from the Cassini-Huygens CAPS electron spectrometer. Planetary and Space Science, 56(7), $901-912$. https://doi.org/10.1016/j.pss.2007.12.017

Ma, X., Delamere, P., Otto, A., \& Burkholder, B. (2017). Plasma transport driven by the three-dimensional Kelvin-Helmholtz instability Journal of Geophysical Research: Space Physics, 122, 10,382-10,395. https://doi.org/10.1002/2017JA024394

Masters, A. (2015). The dayside reconnection voltage applied to Saturn's magnetosphere. Geophysical Research Letters, 42, 2577-2585. https://doi.org/10.1002/2015GL063361

McAndrews, H. J., Thomsen, M. F., Arridge, C. S., Jackman, C. M., Wilson, R. J., Henderson, M. G., et al. (2009). Plasma in Saturn's nightside magnetosphere and the implications for global circulation. Planetary and Space Science, 57(14-15), 1-9. https://doi.org/10.1016/j.pss.2009.03.003

Mitchell, D. G., Brandt, P. C., Roelof, E. C., Dandouras, J., Krimigis, S. M., Mauk, B. H., et al. (2005). Energetic ion acceleration in Saturn's magnetotail: Substorms at Saturn? Geophysical Research Letters, 32, L20S01. https://doi.org/10.1029/2005GL022647

Mitchell, D. G., Kurth, W. S., Hospodarsky, G. B., Krupp, N., Saur, J., Mauk, B. H., et al. (2009). Ion conics and electron beams associated with auroral processes on Saturn. Journal of Geophysical Research, 114, A02212. https://doi.org/10.1029/2008JA013621

Mutel, R. L., Menietti, J. D., Gurnett, D. A., Kurth, W., Schippers, P., Lynch, C., et al. (2010). CMI growth rates for Saturnian kilometric radiation. Geophysical Research Letters, 37, L19105. https://doi.org/10.1029/2010GL044940

Nagai, T., Shinohara, I., Fujimoto, M., Hoshino, M., Saito, Y., Machida, S., \& Mukai, T. (2001). Geotail observations of the Hall current system: Evidence of magnetic reconnection in the magnetotail. Journal of Geophysical Research, 106(A11), 25,929-25,949. https://doi.org/10.1029/2001JA900038

Nakamura, R., Baumjohann, W., Klecker, B., Bogdanova, Y., Balogh, A., Rème, H., et al. (2002). Motion of the dipolarization front during a flow burst event observed by Cluster. Geophysical Research Letters, 29(20), 1942. https://doi.org/10.1029/2002GL015763

Nichols, J. D., Badman, S. V., Baines, K. H., Brown, R. H., Bunce, E. J., Clarke, J. T., et al. (2014). Dynamic auroral storms on Saturn as observed by the Hubble space telescope. Geophysical Research Letters, 41, 3323-3330. https://doi.org/10.1002/2014GL060186 
Ohtani, S. I., Shay, M. A., \& Mukai, T. (2004). Temporal structure of the fast convective flow in the plasma sheet: Comparison between observations and two-fluid simulations. Journal of Geophysical Research, 109, A03210. https://doi.org/10.1029/2003JA010002

Øieroset, M., Phan, T. D., Fujimoto, M., Lin, R. P., \& Lepping, R. P. (2001). In situ detection of collisionless reconnection in the Earth's magnetotail. Nature, 412(6845), 414-417. https://doi.org/10.1038/35086520

Pan, Q., Ashour-Abdalla, M., Walker, R. J., \& El-Alaoui, M. (2014). lon energization and transport associated with magnetic dipolarizations. Geophysical Research Letters, 41, 5717-5726. https://doi.org/10.1002/2014GL061209

Phan, T. D., Shay, M. A., Gosling, J. T., Fujimoto, M., Drake, J. F., Paschmann, G., et al. (2013). Electron bulk heating in magnetic reconnection at Earth's magnetopause: Dependence on the inflow Alfvén speed and magnetic shear. Geophysical Research Letters, 40, 4475-4480. https://doi.org/10.1002/grl.50917

Reed, J. J., Jackman, C. M., Lamy, L., Kurth, W. S., \& Whiter, D. K. (2018). Low frequency extensions of the Saturn kilometric radiation as a proxy for magnetospheric dynamics. Journal of Geophysical Research: Space Physics, 123, 443-463. https://doi.org/10.1002/2017JA024499

Runov, A., Angelopoulos, V., Sitnov, M. I., Sergeev, V. A., Bonnell, J., McFadden, J. P., et al. (2009). THEMIS observations of an earthward-propagating dipolarization front. Geophysical Research Letters, 36, L14106. https://doi.org/10.1029/2009GL038980

Russell, C. T., Jackman, C. M., Wei, H. Y., Bertucci, C., \& Dougherty, M. K. (2008). Titan's influence on Saturnian substorm occurrence. Geophysical Research Letters, 35, L12105. https://doi.org/10.1029/2008GL034080

Smith, A. W., Jackman, C. M., \& Thomsen, M. F. (2016). Magnetic reconnection in Saturn's magnetotail: A comprehensive magnetic field survey. Journal of Geophysical Research: Space Physics, 121, 2984-3005. https://doi.org/10.1002/2015JA022005

Smith, A. W., Jackman, C. M., Thomsen, M. F., Sergis, N., Mitchell, D. G., \& Roussos, E. (2018). Dipolarization fronts with associated energized electrons in Saturn's magnetotail. Journal of Geophysical Research: Space Physics, 123, 2714-2735. https://doi.org/10.1002/2017JA024904

Tao, C., Lamy, L., \& Prangè, R. (2014). The brightness ratio of H Lyman- $\alpha / H_{2}$ bands in FUV auroral emissions: A diagnosis for the energy of precipitating electrons and associated magnetospheric acceleration processes applied to Saturn. Geophysical Research Letters, 41 6644-6651. https://doi.org/10.1002/2014GL061329

Thomsen, M. F., Wilson, R. J., Tokar, R. L., Reisenfeld, D. B., \& Jackman, C. M. (2013). Cassini/CAPS observations of duskside tail dynamics at Saturn. Journal of Geophysical Research: Space Physics, 118, 5767-5781. https://doi.org/10.1002/jgra.50552

Thomsen, M. F., Jackman, C. M., Mitchell, D. G., Hospodarsky, G., Kurth, W. S., \& Hansen, K. C. (2015). Sustained lobe reconnection in Saturn's magnetotail. Journal of Geophysical Research: Space Physics, 120, 10,257-10,274. https://doi.org/10.1002/2015JA021768

Thomsen, M. F., Jackman, C. M., Tokar, R. L., \& Wilson, R. J. (2014). Plasma flows in Saturn's nightside magnetosphere. Journal of Geophysical Research: Space Physics, 119, 4521-4535. https://doi.org/10.1002/2014JA019912

Thomsen, M. F., Reisenfeld, D. B., Wilson, R. J., Andriopoulou, M., Crary, F. J., Hospodarsky, G. B., et al. (2014). Ion composition in interchange injection events in Saturn's magnetosphere. Journal of Geophysical Research: Space Physics, 119, 9761-9772. https://doi.org/10.1002/2014JA020489

Vasyliunas, V. M. (1983). Plasma distribution and flow, physics of the Jovian magnetosphere (pp. 395-453). New York: Cambridge University Press. https://doi.org/10.1029/2003JD004173.Aires

Vogt, M. F., Kivelson, M. G., Khurana, K. K., Joy, S. P., \& Walker, R. J. (2010). Reconnection and flows in the Jovian magnetotail as inferred from magnetometer observations. Journal of Geophysical Research, 115, A06219. https://doi.org/10.1029/2009JA015098

Woch, J., Krupp, N., \& Lagg, A. (2002). Particle bursts in the Jovian magnetosphere: Evidence for a near-Jupiter neutral line. Geophysical Research Letters, 29(7), 1138. https://doi.org/10.1029/2001GL014080

Yao, Z. H., Grodent, D., Ray, L. C., Rae, I. J., Coates, A. J., Pu, Z. Y., et al. (2017). Two fundamentally different drivers of dipolarizations at Saturn. Journal of Geophysical Research: Space Physics, 122, 4348-4356. https://doi.org/10.1002/2017JA024060

Young, D. T., Berthelier, J. J., Blanc, M., Burch, J. L., Coates, A. J., Goldstein, R., et al. (2004). Cassini plasma spectrometer investigation. Space Science Reviews, 114(1-4), 1-112. https://doi.org/10.1007/s11214-004-1406-4

Zong, Q.-G., Fritz, T. A., Pu, Z. Y., Fu, S. Y., Baker, D. N., Zhang, H., et al. (2004). Cluster observations of earthward flowing plasmoid in the tail. Geophysical Research Letters, 31, L18803. https://doi.org/10.1029/2004GL020692 